\title{
O panorama das imunizações no Brasil: um estudo epidemiológico no período de
} 2017 a 2020

\section{The panorama of immunizations in Brazil: an epidemiological study in the period from 2017 to 2020}

\author{
Mariana de Sousa Meneses Carvalho \\ Instituição: Centro Universitário Uninovafapi (UNINOVAFAPI), Teresina-Piauí. \\ Lorena Karen de Morais Moura Castro \\ Instituição: Centro Universitário Uninovafapi (UNINOVAFAPI), Teresina-Piauí. \\ Milena Viana Freire \\ Instituição: Centro Universitário Uninovafapi (UNINOVAFAPI), Teresina-Piauí. \\ Ycaro Kylldary Macêdo \\ Universidade Federal do Piauí (UFPI), Teresina-Piauí. \\ Aureliano Machado de Oliveira \\ Instituição: Centro Universitário Uninovafapi (UNINOVAFAPI), Teresina-Piauí.
}

\begin{abstract}
RESUMO
A imunização é o método terapêutico que visa a proteção imunológica contra doenças infecciosas. Tratase da forma mais efetiva para o controle, a erradicação, e até a eliminação de determinadas enfermidades, o que evidencia a necessidade de maior adesão a essa prática.
\end{abstract}

Palavras-chave: Imunização, Vacina, Imunobiológicos.

\begin{abstract}
Immunization is the therapeutic method that aims the immunological protection against infectious diseases. It is the most effective way to control, eradicate, and even eliminate certain diseases, which highlights the need for greater adherence to this practice.
\end{abstract}

Keywords: Immunization, Vaccine, Immunobiologicals.

\section{INTRODUÇÃO}

O Programa Nacional de Imunizações (PNI), criado em 1973, é determinante para o controle bemsucedido das doenças imunopreveníveis no Brasil (DOMINGUES CMAS, et al., 2019). Sua atuação garante melhorias importantes no Sistema de Saúde do país, visto que, através da vacinação, o Brasil erradicou doenças de alcance mundial como a poliomielite.

O Brasil foi pioneiro na incorporação de diversas vacinas no calendário do Sistema Único de Saúde (SUS) e é um dos poucos países que ofertam, de maneira universal e gratuita, um rol extenso de imunobiológicos (CRUZ A, 2017). 
A garantia da segurança do processo de produção, distribuição, armazenamento e aplicação das vacinas é o principal objetivo do programa "Vacina Segura", institucionalizado pelo PNI (OLIVEIRA VCD, et al., 2019). Implementar medidas como essas é de grande importância, pois também visa mitigar os movimentos antivacinais, cada vez mais crescentes (SATO APS, 2018).

A vacinação proporciona imunidade individual e coletiva. Com a imunização, é possível garantir que doenças erradicadas não retornem e a proteção em massa acontece somente quando $80 \%$ da população é imunizada, a "imunidade em rebanho" (ALVES SMC, et al., 2020). Dessa forma, compreender as variáveis do processo de imunização é fundamental para a melhoria da Saúde Pública brasileira.

\section{OBJETIVO}

Analisar o panorama das imunizações no Brasil, durante o período de janeiro de 2017 a dezembro de 2020, bem como suas principais variáveis e suas implicações na Saúde Pública brasileira.

\section{MÉTODO}

Consiste em um estudo epidemiológico descritivo, com abordagem quantitativa, do tipo retrospectivo. A coleta de dados foi realizada mediante ao uso de registros disponibilizados no Sistema de Informação em Saúde (SIS), referentes ao período de 2017 a 2020 e disponíveis no Departamento de Informática do SUS (DATASUS). Em se tratando de pesquisa com base de dados de acesso público, não se fez necessária a submissão ao Comitê de Ética em Pesquisa (CEP). Foram analisadas as seguintes variáveis em relação à imunização: taxa de abandono, cobertura vacinal, região, imunobiológicos e doses aplicadas. As informações coletadas foram exportadas e tabuladas no software Microsoft Office Excel 2019 para uma melhor análise e discussão.

\section{RESULTADOS}

Foram contabilizadas 441.888.424 doses de imunobiológicos aplicadas no período de 2017 a 2020 no Brasil. As quais tiveram maior concentração na região sudeste (195.524.574) e menor concentração na região centro-oeste (33.361.405).

Ainda referente às doses aplicadas, é possível observar que em 2020 foi aplicada a menor quantidade de doses (99.729.147) em relação aos demais anos avaliados. Foram identificados 23 imunobiológicos, entre os quais destacam-se as vacinas: BCG-ID, Hepatite B, Tetravalente e Pneumocócica.

Quanto ao percentual de vacinados que iniciaram o esquema vacinal e não finalizaram, é possível perceber que o ano de 2019 foi marcado por maior taxa de abandono, totalizando 21,60\%, seguido pelos anos de $2020(18,49 \%)$ e de $2017(16,42 \%)$. 
No Gráfico 1, nota-se um elevado declínio da cobertura vacinal ao longo dos anos, sendo que o ano de 2020 obteve o menor valor, totalizando 66,56\%. Ademais, a região Norte foi a de menor cobertura, seguida da região Nordeste.

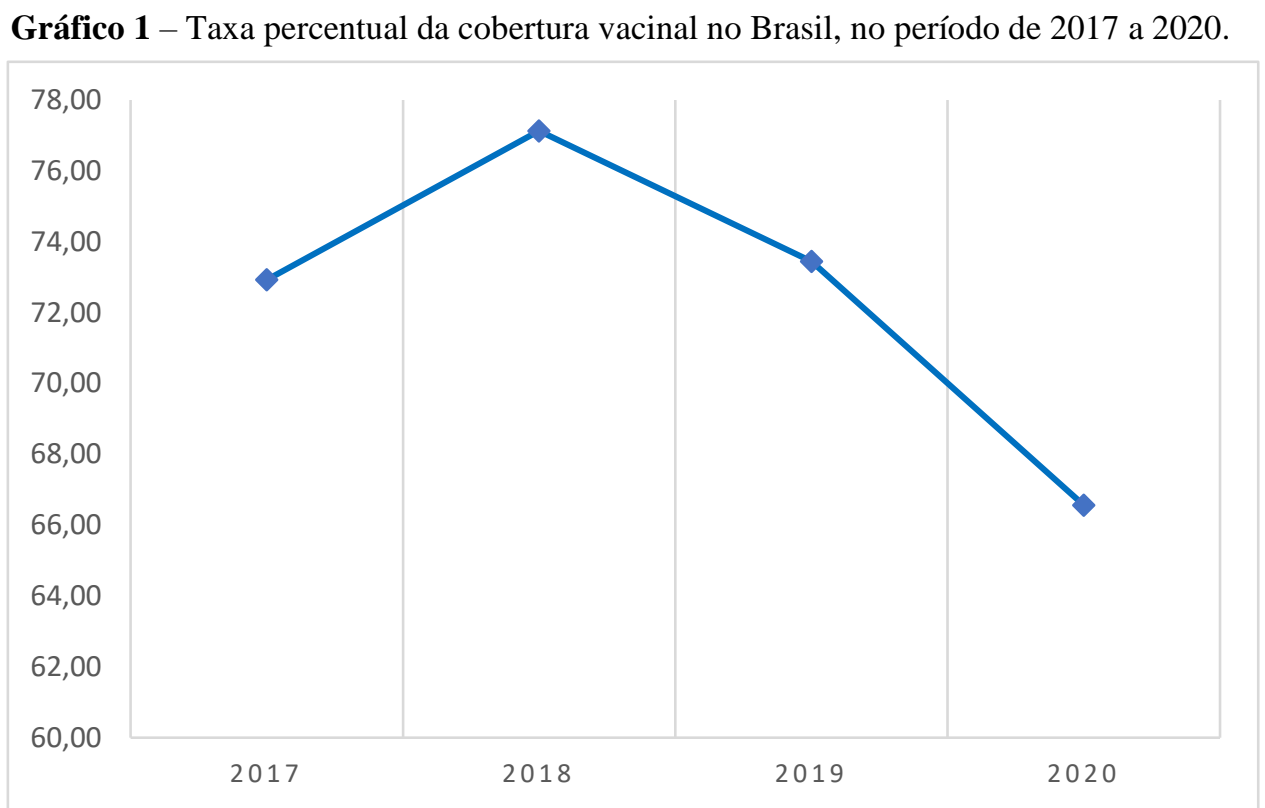

Fonte: CARVALHO MSM, et al., 2021. [Dados extraídos do DATASUS, 2021].

\section{DISCUSSÃO}

De fato, com a diminuição das doses aplicadas e da cobertura vacinal, a imunização, no Brasil, reduziu significativamente. Logo, há a criação de "bolsões de pessoas suscetíveis" a doenças que já foram erradicadas no país (CRUZ A, 2017).

Os novos surtos de doenças imunopreviníveis, que haviam sido controladas, é preocupante para a Saúde Pública brasileira. Como exemplo tem-se o surto de Sarampo - ocorrido em 2018 - com ênfase na região Norte (região de menor cobertura vacinal). Esse resultado está relacionado, também, ao crescimento do movimento antivacina, que questiona a segurança dos imunobiológicos e seus possíveis efeitos colaterais (FRANCO MAE, et al., 2020).

A crescente taxa de abandono reflete a baixa adesão ao programa de vacinação. $O$ percentual significativo das regiões Norte e Nordeste concorda com evidências em que as menores adesões podem resultar de baixas condições socioeconômicas e das dificuldades no acesso à vacina (FERREIRA AV, et al., 2017).

\section{CONSIDERAÇÕES FINAIS}

Conforme a análise epidemiológica acerca das imunizações, compreende-se tamanha importância do programa de vacinação para a Saúde Pública brasileira e, concomitantemente, alerta sobre a redução da 
cobertura vacinal e o aumento da taxa de abandono, sobretudo nas regiões Norte e Nordeste. Dessa forma, há uma enorme necessidade de implementar estratégias para envolver a população nas ações de imunização, propiciando informação e conhecimento, além de reduzir as barreiras para a vacinação. Ainda, o contínuo progresso das campanhas de imunização, junto à vigilância epidemiológica devem ser fomentados, de modo a garantir a cobertura de vacinação em território nacional.

\section{REFERÊNCIAS}

1. ALVES SMC, et al. Vacinação: direito individual ou coletivo? Cad. Ibero-amer., 2020; 9(3): 134-139.

2. CRUZ A. A queda da imunização no Brasil. Revista Consensus, 2017; 25: 1-29.

3. DOMINGUES CMAS, et al. Vacinas Brasil e estratégias de formação e desenvolvimento em imunizações. Epidemiologia e Serviços de Saúde, 2019; 28: e000200024.

4. FERREIRA AV, et al. Acesso à sala de vacinas da Estratégia Saúde da Família: aspectos organizacionais. Rev Enferm UFPE on line, 2017; 94: 574-581.

5. FRANCO MAE, et al. Causas da queda progressiva das taxas de vacinação da poliomielite no Brasil. Brazilian Journal of Health Review, 2020; 3(6): 18476-18486.

6. MIZUTA AH, et al. Percepção acerca da importância das vacinas e da recusa vacinal numa escola de medicina. Rev. Paul. Pediatr., 2019; 37(1): 34-40.

7. OLIVEIRA VCD, et al. A percepção da equipe de enfermagem sobre a segurança do paciente em sala de vacinação. Ver. Cuidarte,2019; 10(1): 11.

8. SATO APS. Qual a importância da hesitação vacinal na queda das coberturas vacinais no Brasil? Rev. Saúde Pública. 2018; 52:96. 